lasted for more than 6 weeks and more than $1 \%$ sustained permanent disability. There were five deaths ( $1 \%$ proportionate mortality), all were due to falls. Few families recounted using seatbelts $(2 \%)$, car air bags $(0.5 \%)$, helmets $(1 \%)$, child restraint seats $(1 \%)$. The majority of children received care via government insurance.

Conclusions The burden of childhood injuries on the university hospital in Egypt is substantial. Ongoing child injury surveillance using systematic approaches is required to identify the epidemiology of injuries, their risk factors and plan for timely interventions.

\title{
0090 CHILDHOOD UNINTENTIONAL INJURIES SURVEILLANCE IN ISMAILIA GOVERNORATE, EGYPT
}

H El-Sayed*, A Hyder, O Zekry, D Sugerman, S Abdel-Hamid, H Abbas, P Puvanachandra Correspondence: Department of Pediatrics, Suez Canal University Hospital, Faculty of Medicine, Suez Canal University, Ismailia, Egypt

10.1136/ip.2010.029215.90

Objective To determine the epidemiology and risk factors of childhood injuries in Ismailia governorate Egypt.

Methods This is the Egyptian chapter of the Global Childhood Unintentional Injury Surveillance study, which employed quota sampling of children 0-11 years old presenting to the Emergency Department. This surveillance was conducted in the University Hospital of Suez Canal University Egypt, for selected months in 2007. Children with an unintentional injury of both genders were enrolled in the study.

Results Out of a total of 551 cases, 361 were males (66\%) and $478(34 \%)$ females. The most common external causes of injuries were falls $(60 \%)$, road traffic injuries $(15 \%)$, burns $(7 \%)$, poisoning (3\%) and near drowning/drowning (2\%). The most common sustained injuries were fractures $(17 \%)$, cuts or open wounds (15\%), sprains (14\%) and burns (9\%). The majority of patients were treated and discharged home without disability $(67 \%)$, while $7.8 \%$ had long-term temporary disability that 